\title{
Aspects Épidémiologiques et Thérapeutiques des Traumatismes Balistiques en Milieu Civil à Cotonou
}

\author{
Pascal Chigblo, \\ Koovi Penance Agbélélé, \\ Service de Traumatologie Orthopédie, CNHU-HKM Cotonou, Bénin \\ François Amossou, \\ Service de Chirurgie, CHUD-OP, Porto-Novo, Bénin \\ Padonou Adebola, \\ Éric Lawson, \\ Iréti Fiacre Tidjani, \\ Soumaïla Madougou, \\ Aristote Hans-Moevi Akue, \\ Service de Traumatologie Orthopédie, CNHU-HKM Cotonou, Bénin
}

\section{Résumé}

Introduction : l'incidence des lésions par balle est en nette augmentation dans nos pays. Le traitement de ces lésions en milieu civil a beaucoup évolué ces dernières années. Le but de cette étude était de décrire les aspects sociodémographiques, lésionnels et thérapeutiques des traumatismes par arme à feu en milieu civil au CNHU-HKM de Cotonou. Matériels et méthodes : Il s'est agi d'une étude rétrospective descriptive menée au Centre National Hospitalier Universitaire Hubert Koutoukou Maga (CNHU-HKM) de Cotonou, et couvrant la période de Janvier 2015 à Décembre 2016 (2 ans). Etaient inclus dans l'étude, les dossiers des blessés par balle, pris en charge dans le service de Traumatologie-Orthopédie et de Chirurgie Réparatrice du CNHU-HKM. Les patients ayant bénéficié d'une prise en charge initiale avant leur admission et ceux agés de moins de 15 ans étaient exclus. Résultats : quinze patients étaient recensés durant la période d'étude. Le délai d'admission médian était de 13 heures. Tous les patients étaient de sexe masculin et l'âge médian était de 33,71 ans. Les circonstances se répartissaient en agression (11 cas, $73,3 \%)$, deux balles perdues $(13,3 \%)$ et deux accidents de manipulation (13,3\%). Dans 9 cas (60\%), une arme de poing était à l'origine des lésions. Une antibioprophylaxie faite d'amoxicilline/acide clavulanique était débutée chez tous les patients et était associée à la gentamicine en cas d'atteinte osseuse. Toutes les lésions avaient été prises en 
charge chirurgicalement selon un protocole comprenant un parage, une exploration et une fermeture primaire différée. Aucune ostéosynthèse interne n'a été effectuée en urgence mais réalisée secondairement après cicatrisation des plaies et négativation de la C-réactive protéine dans un délai médian de 23 jours. Conclusion : les lésions issues des traumatismes balistiques en milieu civil sont rarement graves et leur prise en charge diffère sur certains points de celle des lésions par arme à feu en situation de guerre.

Mots clés: Traumatisme balistique, Milieu civil, Cotonou

\title{
Epidemiological and Therapeutic Aspects of Ballistic Injuries in Civilian Areas in Cotonou
}

\author{
Pascal Chigblo, \\ Koovi Penance Agbélélé, \\ Service de Traumatologie Orthopédie, CNHU-HKM Cotonou, Bénin \\ François Amossou, \\ Service de Chirurgie, CHUD-OP, Porto-Novo, Bénin \\ Padonou Adebola, \\ Éric Lawson, \\ Iréti Fiacre Tidjani, \\ Soumaïla Madougou, \\ Aristote Hans-Moevi Akue, \\ Service de Traumatologie Orthopédie, CNHU-HKM Cotonou, Bénin
}

\begin{abstract}
Introduction: the incidence of gunshot wounds is clearly increasing in our countries. The treatment of these lesions in a civilian environment has evolved considerably in recent years. The aim of this study was to describe the sociodemographic, lesional and therapeutic aspects of civilian firearm trauma at the National Teaching Hospital of Cotonou. Materials and methods: It was a descriptive retrospective study conducted at the National Teaching Hospital of Cotonou, covering the period from January 2015 to December 2016 (2 years). Included in the study were gunshot wounds, supported by Trauma Orthopedic departement. Patients who received initial care before admission and those aged less than 15 years were excluded. Results: fifteen patients were identified during the study period. The median admission time was 13 hours.
\end{abstract}


All patients were male and the median age was 33.71 years. The circumstances were divided into aggression (11 cases, 73.3\%), two lost balls (13.3\%) and two handling accidents (13.3\%). In 9 cases $(60 \%)$, a handgun was causing the injuries. An antibiotic prophylaxis made of amoxicillin / clavulanic acid was started in all patients and was associated with gentamicin in case of bone involvement. All lesions were surgically treated according to a protocol including trimming, exploration and delayed primary closure. No internal fixation was performed urgently but performed secondarily after healing of wounds and negativation of the C-reactive protein in a median time of 23 days. Conclusion: lesions resulting from ballistic injuries in civilian environments are rarely serious and their management differs in some respects from that of firearm injuries in war situations.

Keywords: Ballistic trauma, Civilian environment, Cotonou

\section{Introduction}

Les pays développés que dans les pays en voie de développement pour des raisons diverses (van Vugt, 2003; Rhee, 1998, Khechimi, 2015). Si le pronostic vital est rarement engagé en milieu civil du fait de la faible vélocité des projectiles en cause, les séquelles fonctionnelles sont fréquentes et importantes (Fabeck, 2017). Les chirurgiens urgentistes et traumatologues doivent se familiariser avec cette donne dont la gestion optimale nécessite la connaissance des notions de balistique. Longtemps calqué sur les principes de la chirurgie de guerre, la prise en charge des traumatismes par arme à feu en pratique civile a évolué ces dernières années grâce notamment aux travaux des chirurgiens américains pour qui la gestion de ces lésions constitue un véritable défi quotidien (Seng, 2013). Le but de cette étude était de décrire les aspects sociodémographiques, lésionnels et thérapeutiques des traumatismes par arme à feu en milieu civil au CNHU/HKM de Cotonou

\section{Patients et méthode}

Il s'est agi d'une étude rétrospective descriptive menée au Centre National Hospitalier Universitaire Hubert Koutoukou Maga (CNHU/HKM) de Cotonou, et couvrant la période de Janvier 2015 à Décembre 2016 (2 ans). Etaient inclus dans l'étude, les dossiers des blessés par balle, pris en charge dans le service de Traumatologie-Orthopédie et de Chirurgie Réparatrice du CNHU/HKM. Les patients ayant bénéficié d'une prise en charge initiale avant leur admission et ceux agés de moins de 15 ans étaient exclus.

Les données ont été recueillies à partir des registres d'hospitalisation et de compte rendu opératoire et des dossiers des malades. Les fractures ouvertes étaient classées selon Gustilo et Anderson (Gustilo, 1976). Les 
paramètres étudiés étaient : l'âge, le sexe, les circonstances du traumatisme, le bilan lésionnel et le traitement.

\section{Résultats}

\section{Aspects sociodémographiques}

Quinze patients, tous de sexe masculin ont été recensés durant la période d'étude. L'âge médian était de 33,71 ans (extrêmes : 19 ans et 57 ans). La tranche d'âge de 25 à 35 ans était la plus représentée (11 cas ; 73,3\%). Les couches socio-professionnelles les plus touchées étaient celles des commerçants (3 cas; 20\%) et des agents des forces de l'ordre (3 cas; 20\%).

\section{Aspects lésionnels}

Les circonstances de survenue des traumatismes étaient : une agression (11 cas ; 73,3\%), une balle perdue ( 2 cas; $13,3 \%$ ) et un accident de manipulation d'arme ( 2 cas ; 13,3\%). Les armes à feu en cause étaient : une arme de poing dans 9 cas $(60 \%)$ et une arme d'épaule dans 6 cas $(40 \%)$ dont 3 cas $(20 \%)$ d'arme artisanale, 2 cas $(13,3 \%)$ d'arme de chasse, et un cas $(6,7 \%)$ d'arme de guerre. Le délai médian d'admission des patients était de 13 heures avec des extrêmes de 30 minutes et 4 jours.

Aucun patient ne présentait une instabilité hémodynamique à l'admission. On dénombrait 16 lésions chez les 15 patients. Les lésions étaient osseuses dans 6 cas et concernaient uniquement les parties molles dans 10 cas. Ces lésions siégeaient aux membres dans 14 cas et au cuir chevelu dans 2 cas. Les lésions osseuses concernaient le fémur dans 4 cas, le tibia dans un cas et les métacarpiens dans un cas. Trois fractures étaient comminutives, deux avec un troisième fragment et une simple. Toutes les fractures étaient ouvertes de type IIIa selon de Gustilo et Anderson. Deux fractures fémorales étaient associées à une atteinte du nerf sciatique. Aucune atteinte d'un axe vasculaire majeur n'était retrouvée.

\section{Aspects thérapeutiques}

Une vaccination et une sérothérapie antitétanique avait été instituée chez tous les patients. Une antibioprophylaxie faite d'amoxicilline/acide clavulanique était débutée chez tous les patients et était associée à la gentamicine en cas d'atteinte osseuse. Toutes les lésions avaient été prises en charge chirurgicalement selon un protocole comprenant un parage, une exploration et une fermeture primaire différée. Aucune ostéosynthèse interne n'avait été effectuée en urgence. Une traction trans-osseuse avait été mise en place après parage des fractures fémorales. Une ostéosynthèse par un fixateur externe avait été faite pour la fracture tibiale ; et une immobilisation par attelle plâtrée pour la fracture des métacarpiens. Un enclouage centromédullaire avait été secondairement effectué dans un délai moyen de 23 jours pour les fractures 
fémorales après cicatrisation des plaies et vérification biologiques de l'absence d'infection par dosage de la C-Reactive Protein. Les caractéristiques épidémiologiques cliniques et thérapeutiques sont résumées dans le tableau ci-dessous.

Tableau I : Caractéristiques sociodémographiques, cliniques et thérapeutiques

\begin{tabular}{|c|c|c|c|c|c|c|}
\hline $\begin{array}{c}\mathbf{N} \\
0\end{array}$ & Age & Profession & $\begin{array}{c}\text { Délai } \\
\text { d'admis } \\
\text { sion } \\
\text { (heure) }\end{array}$ & $\begin{array}{c}\text { Type } \\
\text { d'arme }\end{array}$ & Bilan lésionnel & $\begin{array}{c}\text { Traitement } \\
\text { chirurgical initial }\end{array}$ \\
\hline 1 & 57 & Commerçant & 8 & $\begin{array}{l}\text { Arme de } \\
\text { guerre }\end{array}$ & $\begin{array}{l}\text { Fracture ouverte type IIIA de GA* de la } \\
\text { diaphyse fémorale gauche } \\
\text { Fracture ouverte type IIIA de GA* des 3è } \\
\text { et } 4 \text { è métacarpiens gauches }\end{array}$ & $\begin{array}{l}\text { Parage, traction } \\
\text { transtibiale, } \\
\text { attelle platrée palmaire }\end{array}$ \\
\hline 2 & 28 & $\begin{array}{l}\text { Cadre } \\
\text { indépendant }\end{array}$ & $1 / 2$ & $\begin{array}{l}\text { Fusil de } \\
\text { chasse }\end{array}$ & $\begin{array}{l}\text { Fracture ouverte type IIIA de GA* de la } \\
\text { diaphyse fémorale droite, avec atteinte du } \\
\text { Nerf ischiatique }\end{array}$ & $\begin{array}{l}\text { Parage, exploration, } \\
\text { traction transtibiale }\end{array}$ \\
\hline 3 & 31 & Ouvrier & 7 & $\begin{array}{l}\text { Arme de } \\
\text { poing }\end{array}$ & Plaie du cuir chevelu & Parage \\
\hline 4 & 32 & Cultivateur & 96 & $\begin{array}{l}\text { Arme } \\
\text { artisanale }\end{array}$ & Criblage du membre thoracique droit & Parage \\
\hline 5 & 25 & $\begin{array}{l}\text { Agent de Force } \\
\text { de l'ordre }\end{array}$ & 7 & $\begin{array}{l}\text { Arme de } \\
\text { poing }\end{array}$ & Plaie du bras droit & Parage \\
\hline 6 & 32 & $\begin{array}{l}\text { Cadre } \\
\text { administratif }\end{array}$ & 8 & $\begin{array}{l}\text { Arme de } \\
\text { poing }\end{array}$ & Plaie de la cuisse droite & Parage \\
\hline 7 & 33 & Ouvrier & 17 & $\begin{array}{c}\text { Arme } \\
\text { artisanale }\end{array}$ & Plaie de la cheville droite & Parage \\
\hline 8 & 19 & Etudiant & 4 & $\begin{array}{l}\text { Arme de } \\
\text { poing }\end{array}$ & $\begin{array}{l}\text { Fracture ouverte type IIIA de GA* du tibia } \\
\text { gauche }\end{array}$ & Parage, exofixation \\
\hline 9 & 30 & Commerçant & 9 & $\begin{array}{l}\text { Fusil de } \\
\text { chasse }\end{array}$ & $\begin{array}{l}\text { Fracture ouverte type IIIA de GA* de la } \\
\text { diaphyse fémorale gauche }\end{array}$ & Parage, traction \\
\hline 10 & 25 & $\begin{array}{l}\text { Conducteur de } \\
\text { taxi moto }\end{array}$ & 13 & $\begin{array}{l}\text { Arme de } \\
\text { poing }\end{array}$ & Plaie non articulaire du genou gauche & Parage \\
\hline 11 & 34 & $\begin{array}{l}\text { Agent de Force } \\
\text { de l'ordre }\end{array}$ & 2 & $\begin{array}{l}\text { Arme de } \\
\text { poing }\end{array}$ & Plaie du cuir chevelu & Parage \\
\hline 12 & 34 & Enseignant & 12 & $\begin{array}{l}\text { Arme de } \\
\text { poing }\end{array}$ & Plaie du pli de l'aine gauche & Parage \\
\hline 13 & 32 & $\begin{array}{l}\text { Agent de Force } \\
\text { de l'ordre }\end{array}$ & 3 & $\begin{array}{l}\text { Arme de } \\
\text { poing }\end{array}$ & $\begin{array}{l}\text { Fracture ouverte type IIIA de GA* de la } \\
\text { diaphyse fémorale droite, avec atteinte du } \\
\text { Nerf ischiatique }\end{array}$ & $\begin{array}{l}\text { Parage, } \\
\text { transtibiale }\end{array}$ \\
\hline 14 & 47 & Fonctionnaire & 6 & $\begin{array}{l}\text { Arme de } \\
\text { poing }\end{array}$ & Plaie de la cuisse gauche & Parage \\
\hline 15 & 44 & Commerçant & 3 & $\begin{array}{l}\text { Arme } \\
\text { artisanale }\end{array}$ & Plaie articulaire du genou droit & Parage, drainage \\
\hline
\end{tabular}

GA* : Gustilo et Anderson

\section{Discussion}

Les blessés par balle en milieu civil sont de plus en plus fréquents dans la plupart des hôpitaux de la région du fait de la recrudescence des conflits régionaux, mais surtout de l'augmentation de la délinquance juvénile dans les grandes agglomérations africaines compte tenue de l'échec des politiques d'éradications de la pauvreté dans la plupart des pays de la sous-région (Onuminya, 2005 ; Dikongue, 2012 ; Abalo, 2016). Dans ce contexte les agressions par vols à main armée représentent la principale circonstance lésionnelle dans notre série comme dans beaucoup d'autres séries ou les réalités socioéconomiques sont superposables (Onuminya, 2005 ; Dikongue, 
2012). Les accidents de manipulation surviennent essentiellement dans la population des forces de sécurité au cours des séances de formation (Akpoto, 2015). La prépondérance masculine s'explique par le fait que les jeunes hommes sont généralement plus audacieux et plus agressif dans la réponse à une menace.

Les traumatismes balistiques en pratique civile sont souvent considérés comme des traumatismes à basse vélocité (utilisation fréquente d'arme de poing comme dans notre série), mais le transfert d'énergie lors de la déformation du projectile au sein du corps peut se révéler intense (Clasper, 2001). Ainsi la prise en charge chirurgicale initiale de toute plaie par balle aussi bien en pratique civile que militaire comprend un débridement, un parage extensif un lavage et une fermeture primaire souvent (Rouvier, 1997; Robinson, 1989). Les principes de stabilisation des fractures par arme à feu en milieu civil ont évolué ces dernières années et dépendent surtout de la vélocité du projectile contrairement aux traumatismes en situation de guerre où il existe toujours une forte contamination (3 à 4 types de bactéries en moyenne) (Seng, 2013). Lorsqu'elles surviennent à faible vélocité ces fractures sont considérées comme des fractures ouvertes Gustilo I ou II avec atteintes modérées des parties molles (Volgas, 2005 ; Stefanopoulos, 2014) et peuvent donc autoriser d'emblée une ostéosynthèse interne avec de bons résultats (Abalo, 2016). Dans le cas de fractures comminutives associées à des lésions extensives des tissus mous dans les traumatismes à haute vélocité (arme de guerre, fusil de chasse), une exofixation (Hildebrand 2004 ; Hollman 1990) avec ou sans une fasciotomie prophylactique est indiquée pour éviter un syndrome des loges (Van Waes, 2013). Comme le montre les résultats, aucune stabilisation immédiate par ostéosynthèse interne n'a été effectuée bien que certains de nos patients auraient pu en bénéficier au vu des critères suscités, ce qui réduirait considérablement le délai d'hospitalisation. Notre attitude s'explique par le fait que le délai de prise en charge de la plupart de nos patients est largement supérieur à 6 heures d'où un risque d'infection plus élevé, bien que selon plusieurs auteurs, un délai de prise en charge long n'augmente pas le risque infectieux dans les fractures balistiques (Schenker, 2012). Les lésions nerveuses périphériques sont fréquentes et sont présentes chez deux de nos patients. Même si le taux de récupération spontanée avoisine les $70 \%$ en 6 à 9 mois dans la littérature (Bruner, 2011), l'attitude à adopter face à celle-ci n'est pas univoque entre ceux qui préconisent une exploration dans les sept jours et d'autres beaucoup plus tardivement après résorption des phénomènes inflammatoires (Volgas, 2005). Dans notre série l'exploration dans les atteintes nerveuses a été systématiquement faite en urgence. L'antibiothérapie recommandée lors de traumatismes balistiques reste controversée (Fabeck, 2017). L'administration d'une céphalosporine de première génération pendant 48 heures possiblement associée à la gentamicine en cas de fracture ou de plaie 
articulaire est nécessaire (Volgas, 2005, Abubaker 2009). Pour les traumatismes à basse énergie, sans fracture, l'antibiothérapie ne semble pas indispensable (Bruner, 2011; Lichte 2010) sauf association avec certains facteurs de risques (Fabeck, 2017). Dans cette série l'amoxicilline/acide clavulanique a été administré chez tous les patients et a été associée à la gentamycine en cas de fracture.

\section{Conclusion}

Les lésions par arme à feu en milieu civil sont peu fréquentes dans un pays comme le nôtre. Elles ne sont pas gravissimes, mais leur prise en charge en revêt certaines particularités que tout chirurgien doit maitriser afin d'adopter une attitude optimale à adopter face à ces lésions. Une étude prospective reprenant ces nouveaux préceptes serait de mise dans notre contexte afin d'en tirer des conclusions.

\section{Conflits d'intérêt : aucun}

\section{References :}

1. Van Vugt AB. (2003). Pitfalls in penetrating trauma. Acta Chir Belg; 103(4):358-63.

2. Rhee PM, Foy H, Kaufmann C, Areola C, Boyle E, Maier RV, Jurkovich G. (1998). Penetrating cardiac injuries: a population-based study. J Trauma; 45(2):366-70.

3. Khechimi M, Maalla R. (2015). Particularités des traumatismes balistiques au niveau des membres - à propos de 12 cas. Chirurgie de la Main. ; 34(6) : 387

4. Fabeck L, Hock N, Goffine J, Ngatchou W. (2017). Notions de balistique et prise en charge des plaies par balle au niveau des membres. Rev Med Brux ; 38 : 474-81

5. Rouvier B, Lenoir B, Rigal S. (1997). Les traumatismes balistiques. Conférences d'actualisation Elsevier, Paris ; 703-716

6. Robinson D, On E, Hadas N, Halperin N, Hofman S, Bol-dur I. (1989) Microbiologic flora contaminating open fractures: itssignificance in the choice of primary antibiotic agents and the likelihood of deep wound infection. J Orthop Trauma; 3:283-6.

7. Seng VS, Masquelet AC. (2013). Management of civilian ballistic fractures. Orthop Traumatol Surg Res ;99(8):953-8

8. Gustilo RB, Anderson JT. (1976). Prevention of infection in the treatment of 125 open fractures of long bones: retrospective and prospective analyses. J Bone Joint Surg Am; 58:453-68.

9. Onuminya JE, Ohwowhiagbese E. (2005). Pattern of civilian gunshot injuries in Irrua, Nigeria. SAJS; 43(4):170-2. 
10. Dikongue DF, Hans Moevi A, Handy Eone D, Bahebeck J. (2012). Les fractures ouvertes par arme à feu en milieu civil : à propos de 35 cas en milieu urbain africain. Orth. Emerg.Afr ; 1(1) :18-9

11. Akpoto YM, A.Abalo A, Gnandi-pio F, Sonhaye L, Tchaou M, Sama HD. (2015). Aspects épidémiologiques des fractures de membres liées à l'exercice de la fonction militaire au Togo. Pan Afr Med J ; 20: 377

12. Clasper J. (2001). The interaction of projectiles with tissues and the management of ballistic fractures. J R Army Med Corps; 147(1): 5261.

13. Volgas DA, Stannard JP, Alonso JE. (2005). Ballistics: a primer for the surgeon. Injury; 36:373-9.

14. Stefanopoulos PK, Hadjigeorgiou GF, Filippakis K, Gyftokostas D. (2014). Gunshot wounds: A review of ballistics related to penetrating trauma. Journal of Acute Disease ; 178-85 doi: 10.1016/S22216189(14)60041-X

15. Abalo A, Walla A, Ayouba G, Dellanh Y, Fortey K, Dossim A. (2016). Internal Fixation of Gunshot Induced Fractures in Civilians: Anatomic and Functional Results of a Standard Protocol at an Urban Trauma Center. Open Journal of Orthopedics. ; 6 : 63-70

16. Hildebrand F, Giannoudis P, Kretteck C, Pape HC. (2004). Damage control: extremities. Injury; 35(7):678-89.

17. Hollmann MW, Horowitz M. (1990). Femoral fractures secondary to low velocity missiles: treatment with delayed intramedullary fixation. J Orthop Trauma; 4(1):64 9.

18. Van Waes OJF, Van Lieshout EMM, Hogendoorn W, Halm JA, Vermeulen J. (2013). Treatment of penetrating trauma of the extremities: ten years' experience at a Dutch level 1 trauma center. Scand J Trauma Resusc Emerg Med;14:21-2

19. Schenker ML, Yannascoli S, Baldwin KD, Ahn J, MehtaS. (2012). Does timing to operative debridement affect infectious complications in open long-bone fractures? A systematic review. J Bone Joint Surg Am; 94:105764.

20. Bruner D, Gustafson CG, Visintainer C. (2011). Ballistic injuries in the emergency department. Emerg Med Pract; 13(12):1-30.

21. Abubaker AO. (2009). Use of Prophylactic Antibiotics in Preventing Infection of Traumatic Injuries. Dental Clinics of North America; 53(4) : 707-15

22. Lichte P, Oberbeck R, Binnebösel M, Wildenauer R, Pape H-C, Kobbe P. (2010). A civilian perspective on ballistic trauma and gunshot injuries. Scand J Trauma Resusc Emerg Med; 17:18-35. 